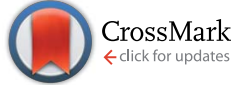

Cite this: J. Anal. At. Spectrom., 2016, 31,1605

Received 22nd January 2016 Accepted 24th May 2016

DOI: $10.1039 / \mathrm{c} 6 \mathrm{ja} 00020 \mathrm{~g}$

www.rsc.org/jaas

\title{
Investigation on the suitability of ablated carbon as an internal standard in laser ablation ICP-MS of polymers $\uparrow$
}

\author{
D. Deiting, ${ }^{\text {*ad }}$ F. Börno, ${ }^{b}$ S. Hanning, ${ }^{a}$ M. Kreyenschmidt, ${ }^{a}$ T. Seidl ${ }^{c}$ and M. Otto ${ }^{d}$
}

The conventional quantitative method for the analysis of inorganic elements in polymer matrices is a complex and time consuming process that presents a significant risk for error. Typically, polymers are digested in a microwave oven or other devices under high temperature and pressure for several hours while employing different mixtures of high purity acids. In many cases, particularly when high concentrations of doped elements are present, the digestion is often incomplete and therefore the reproducibility depends strongly on the type of polymer and additives used. A promising alternative technology that allows for the direct analysis of these polymers without digestion is laser ablation ICP-MS. Due to a lack of available reference materials and the presence of matrix dependent effects, a precise calibration cannot be obtained. In order to compensate for the matrix dependent effects the use of internal standardization is necessary. In this study the correlation between the carbon released during the ablation process and the ${ }^{13} \mathrm{C}$ signal detected by ICP-MS and its use as an internal standard are investigated. For this purpose, twenty-one virgin polymer materials are ablated; the released carbon is determined and correlated with the corresponding integrated ${ }^{13} \mathrm{C}$ signal. The correlation resulted in a direct relationship between the ablated carbon and ${ }^{13} \mathrm{C}$ signal demonstrating the potential ability to neglect at least some of the matrix dependent and transport effects which occur during the laser ablation of virgin polymers.

\section{Introduction}

Plastics are widely applicable in numerous industrial fields such as the electronic sector, automotive industry, packaging of goods and the building sector. The successful implementation of plastics into almost all aspects of modern life was achieved through the introduction of a massive variety of organic and inorganic substances, known as additives, into the virgin polymer matrix. These additives are applied in order to extend or tailor specific material properties such as elasticity, color, hardness, flame- and UV resistance etc. Due to the discovery of their potentially harmful influence on consumers' health and the natural environment ${ }^{\mathbf{1 , 2}}$ the utilization of certain additives became restricted by law (e.g. 2011/65/EU RoHS, 2002/96/EG

${ }^{a}$ University of Applied Sciences Münster, Department of Chemical Engineering, Advanced Analytical Chemistry, Stegerwaldstr. 39, 48565 Steinfurt, Germany. E-mail: d.deiting@fh-muenster.de

${ }^{b} B A M$ Federal Institute for Material Research and Testing, Richard-Willstaetter-Str.11, 12489 Berlin, Germany

${ }^{c}$ Westphalian Institute for Biomimetics, Westphalian University of Applied Sciences, Münsterstr. 265, 46397 Bocholt, Germany

${ }^{d}$ TU Bergakademie Freiberg, Institute of Analytical Chemistry, Leipziger Str. 29, 09599 Freiberg, Germany

$\dagger$ Electronic supplementary information (ESI) available. See DOI: 10.1039/c6ja00020g
WEEE, 2009/48/EC safety of toys). The control of these regulated elements is a challenging task due to the huge variety of different plastics with varying elemental compositions. One suitable technique for the quantitative determination of regulated elements in polymer based materials is the inductively coupled plasma mass spectrometry (ICP-MS). ${ }^{3}$ The advantages of this technology include excellent limits of detection, multielement capability and high throughput. Unfortunately, in most cases sample preparation that involves the digestion of the polymer employing concentrated mineral acids and pressure is required before the measurements can be performed. Closed vessel microwave digestion and high pressure ashing are techniques often applied in order to destroy the polymer matrix and liquefy the inorganic elements. These preparation methods, while very reliable for a large variety of samples, suffer from several well-known significant drawbacks with regard to the analysis of plastics. ${ }^{4-7}$

The use of laser ablation (LA) as a direct sample introduction technique in ICP enables a significant reduction in the sample preparation steps required. ${ }^{8-10}$ However this technique also exhibits several disadvantages. The major problems arise from elemental fractionation ${ }^{\mathbf{9 1 1}, 12}$ such as matrix and transport effects. ${ }^{13-16}$ These factors restrict the routine use of this technique for the quantitative determination of elements in plastics. 
Differences in the physical properties and UV absorption characteristics of varying polymer classes strongly influence the ablation efficiencies, which therefore results in different ablation rates. ${ }^{17}$ One approach in order to correct the different ablation efficiencies of varying polymers is to use the carbon ${ }^{13} \mathrm{C}$ isotope signal as an internal standard. ${ }^{18-23}$ Carbon is primarily chosen because it is the major element present in all polymer based materials and is homogeneously distributed throughout the polymer matrix.

One of the major limitations that impacts the suitability of ${ }^{13} \mathrm{C}$ as an internal standard arises from the formation of different products in the ablation process of different polymer types. ${ }^{15,24,25}$ The deposition of the carbon containing material onto the sample surface during the laser ablation of organic materials appears to also limit this approach. ${ }^{26,27}$ Analytical investigation indicates that the type of carbon ablation products depends on the sample as well as the ablation gas used. ${ }^{24,26,28}$ Investigation into carbon-containing gaseous species and carbon-containing particle phases elucidated a matrix dependent behavior concerning the amount of carbon-containing gaseous species. ${ }^{24}$

Despite the elemental fractionation, the suitability of ${ }^{13} \mathrm{C}$ carbon to compensate for variations in the ablated mass and mass transport efficiencies was previously reported. Austin et al. used the ${ }^{13} \mathrm{C}$ signal for the correction of these effects for the ablation of chicken breast tissue. ${ }^{19}$ Becker et al. corrected the water content in tobacco leaves using ${ }^{13} \mathrm{C}$ as an internal standard. ${ }^{29}$ The determination of silver in different polymers resulted in good accordance of measured and expected values. A polypropylene sample was chosen as a standard and the silver contents of other samples consisting of polypropylene, acrylonitrile-butadiene-styrene and nitrile butadiene rubber were determined using ${ }^{13} \mathrm{C}$ as an internal standard. Only the measurement of silver in polyoxymethylene provided results four times higher than that expected. ${ }^{21}$

The laser ablation studies performed thus far focused only on a small range of polymers and other organic materials. A wide variety of polymers with different structures, functional groups and atoms are commonly used. Due to the varying compositions the polymers possess different material properties which affect the behavior in the laser ablation process.

General investigation regarding the influence of the polymer structures on the ablation process for analytical purposes has not been conducted until now. In order to utilize ${ }^{13} \mathrm{C}$ as an internal standard in routine applications, a correlation between the ${ }^{13} \mathrm{C}$-ICP-MS signals of different polymers must be present. It is necessary to assure this relationship before a possible quantification using ${ }^{13} \mathrm{C}$ as the internal standard can be performed. Only in the case of an established coherence might a correction utilizing the ${ }^{13} \mathrm{C}$ signal be feasible. Therefore, the influence of several parameters and the applicability of ${ }^{13} \mathrm{C}$ correction must be studied in further detail.

In this study the correlation between ablation crater volumes of twenty-one virgin polymers and the resulting ${ }^{13} \mathrm{C}$-ICP-MS signals was evaluated. The ablation crater volumes were measured and the calculated ablated carbon was correlated with the corresponding ${ }^{13} \mathrm{C}$ ICP-MS signal.

\section{Experimental}

As the laser ablation system a LSX-213 (CETAC, Omaha, USA) equipped with a Nd:YAG laser with a fixed wavelength of $213 \mathrm{~nm}$ and an in-house designed ablation cell was employed. ICP-MS measurements were conducted using an ELAN DRC II (Perkin Elmer Sciex, Rodgau, Germany). The utilized laser and ICP-MS parameters are given in Table 1.

ICP-MS and laser parameters were optimized in advance by means of an in-house produced LDPE standard. The general production of these standards can be found elsewhere. ${ }^{30}$ For optimization single line scans were applied since a drop of signal intensity over time for single point measurements was observed.

For line scan measurements, the scan rate was $25 \mu \mathrm{m} \mathrm{s}^{-1}$. Nebulizer gas flow, ICP RF power, ion lens, cell path voltage, ablation gas flow and pulse repetition rate were optimized to attain the highest ${ }^{13} \mathrm{C}$ signal intensity. The laser ablation system used limited the pulse repetition rate to $20 \mathrm{~Hz}$. This pulse repetition rate resulted in the highest signal intensity. The inhouse built ablation cell consists of a cylindrical cavity with a cell volume of $4.75 \mathrm{~cm}^{3}$, one gas inlet and one gas outlet. Due to the low cell volume a maximum ablation gas flow of $200 \mathrm{~mL} \mathrm{~min}^{-1}$ was applied. The nebulizer gas flow of the ICP-MS is added to the ablation gas behind the ablation cell which allows for sample exchange without influencing the plasma stability.

The laser pulse energy of $0.6 \mathrm{~mJ}$ enabled good sensitivities. Higher values led to a high amount of carbon containing material being deposited on the ablation cell window. The laser pulse energy was determined with a pyroelectric energy meter Orion-PE PE25BF-V2 (Ophir Optronics Solutions Ltd, Har Hitzvim, Israel).

Subsequent to LA-ICP-MS measurements, the polymer surfaces as well as the ablation crater volumes and ablation crater shapes of each ablation crater were measured with a confocal microscope $\mu$-surf basic (NanoFocus AG, Oberhausen, Germany). The software $\mu$ soft analysis automatically calculates the ablation crater volumes as well as the maximum depth of the analyzed ablation crater. For best results the highest resolution of 50 -fold magnification and height increments of $48 \mathrm{~nm}$ were chosen.

Table 1 Laser and ICP-MS parameters

\section{Laser parameters}

Ablation mode

Laser pulse energy

Pulse repetition rate

Number of shots per point

Spot size

Ablation gas flow

\section{ICP-MS parameters}

Nebulizer gas flow

Auxiliary gas flow

Outer gas flow

ICP RF power

Dwell time
Single point measurement

$0.6 \mathrm{~mJ}$

$20 \mathrm{~Hz}$

$100 / 50$

$200 \mu \mathrm{m}$

$200 \mathrm{~mL} \mathrm{~min}^{-1}$

$1.26 \mathrm{~L} \mathrm{~min}^{-1}$
$1 \mathrm{~L} \mathrm{~min}^{-1}$
$15 \mathrm{~L} \mathrm{~min}^{-1}$
$1250 \mathrm{~W}$
$50 \mathrm{~ms}$


A total amount of twenty-one different virgin, undoped polymers were analyzed during this study. A detailed description of the polymers used in this study is provided in the ESI. $\dagger$

The investigated polymers contained the classes polyolefins, polyamides, polyesters, polyethers, polyacetals, styrene containing polymers and polysulfones. In order to obtain even polymer surfaces, which are favorable to the laser ablation and confocal microscope measurements, the granulates of sixteen polymers were heated and pressed into round discs with a diameter of $40 \mathrm{~mm}$ using a mounting press (SimpliMed 3000, Buehler, Duesseldorf, Germany). Due to the high melting points of some investigated polymers, or the beginning of decomposition at higher temperatures, the production of plates was not possible for all polymers with the available laboratory equipment. Therefore, pellets of the polymers (polyethersulfone (PES), polyamide 6 and 66 (PA 6 and PA 66), poly(vinyl chloride) (PVC), and polyphthalamide (PPA)) were ablated. Due to the uneven surface, the precise determination of the exact ablation crater volumes for these polymers was restricted.

LA-ICP-MS experiments were initially conducted using ten polymers in order to determine the best ablation parameters for measuring the ablation crater volumes. These polymers included acrylonitrile-butadiene-styrene (ABS), polystyrene (PS), polycarbonate (PC), polyethylene terephthalate (PET), polyamide 12 (PA 12), polyamide 6/12 (PA 6/12), polyoxymethylene (POM), polyether ether ketone (PEEK), polyethersulfone (PES) and polysulfone (PSU). Five single point measurements with 50, 100 and 200 laser shots each were applied to the mentioned samples. These measurements were performed using $\mathrm{He}$ as the carrier gas with a flow of $200 \mathrm{~mL} \mathrm{~min}^{-1}$. In order to fit into the in-house ablation cell, the samples were cut down to a size of about $5 \mathrm{~mm} \times 5 \mathrm{~mm}$ using a knife.

Later on five single point measurements with 100 laser shots each were carried out on defined positions of all twenty-one polymer samples. With a distance of $500 \mu \mathrm{m}$ between the spots, five ablation spots were applied in a row at the center of the ablation cell along the $x$-axis.

The total mass of ablated carbon was calculated using the ablated volume, the density of the polymers and the amount of carbon within the polymers.

The densities of the polymer discs were calculated using the heights, diameters and masses of the samples. The density determinations of the polymer pellets were conducted using a pycnometer (BRAND GmbH \& Co KG, Wertheim, Germany). The amount of carbon was determined using VARIO Macro (Elementar Analysensysteme $\mathrm{GmbH}$, Hanau, Germany) at Federal Institute for Materials Research and Testing (BAM, Berlin, Germany).

The measured ${ }^{13} \mathrm{C}$-ICP-MS signals of all polymers in each experiment were integrated over time. The mean value and the corresponding standard deviation of five single measurements of the ${ }^{13} \mathrm{C}$ signal were calculated. The gas blank was subtracted from the mean. Afterwards, the integrated ${ }^{13} \mathrm{C}$ signals were correlated with the total mass of ablated carbon of the corresponding polymers.
Microtome sections of $10 \mu \mathrm{m}$ thickness were prepared (Leitz 1400 sledge microtome, Leica Microsystems, Wetzlar, Germany) and UV/VIS spectra were recorded from PA 12, LDPE, HDPE, ABS and HIPS in the range from 190 to $400 \mathrm{~nm}$ with an UV/VIS spectrometer (Specord210, Analytik Jena AG, Jena, Germany) to specify the ablation characteristics of different polymers. For other polymers, the preparation of microtome sections with $10 \mu \mathrm{m}$ thickness was not possible due to their brittleness. UV-VIS measurements were not possible for microtome sections with thicknesses exceeding $10 \mu \mathrm{m}$.

\section{Results and discussion}

\section{General findings regarding ablation crater rims, shapes and volumes}

To determine the correlation between the measured ${ }^{13} \mathrm{C}$-ICP-MS signal and the ablated carbon of the samples it was necessary to determine the ablation crater volume. Confocal microscope measurements were performed to evaluate the influence of the laser on the ablation crater volume as well as on the shape of ablation craters of different polymers.

These measurements resulted in figures showing elevation profiles. The crater bottoms, which are the deepest parts of the samples, are depicted in a dark blue color, while the polymer surfaces, the highest parts of the samples, are depicted in a light rose color as can be seen in Fig. 1. Colors between dark blue and light rose reflect the walls of the ablation crater and give information about the shape (cylindrical or conical) of the ablation craters.

The conducted confocal microscope measurements showed a dependency of the topology of the ablation crater on the type of polymer applied. Most pronounced conical ablation craters were observed for LDPE, HDPE, POM and PP, while most pronounced cylindrical ablation craters were observed for the polyamides. The other polymers exhibited primarily conical shaped ablation craters without flat bottoms (Fig. 1). Noncylindrical ablation craters have been found before for glass and polymer samples..$^{31-33}$

The attained results regarding the ablation crater appearances of different virgin polymers lead to the conclusion that the polymeric backbone has an important influence on the ablation behavior. Those polymers whose topologies diverge

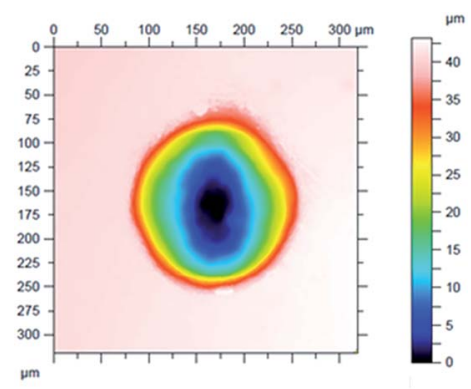

Fig. 1 Predominant ablation crater shape for most polymers (ABS 100 laser shots). 
most from the cylindrical shaped ablation craters are the polyolefins.

The structures and related properties of polyolefins differ from those of most other polymers used in this study. Polyolefins are composed only of carbon and hydrogen, while POM also contains oxygen in the backbone.

The structures of polymers have an important influence on the absorption of UV light due to their different functional groups. ${ }^{17,34-36}$ The difference in the structures leads to a less pronounced absorption of UV light by the polyolefins when compared to other polymers with strongly absorbing functional groups in their structures like ABS or the polyamides. In Fig. 2 the UV/VIS spectra show stronger absorption of virgin ABS and virgin PA 12 compared to virgin LDPE. The absorption of ABS and PA 12 especially in the range from 190 to $225 \mathrm{~nm}$ is too strong to perform reliable measurements. Nevertheless it can be stated that the UV absorption in this range is stronger for these two polymers compared to LDPE. The UV/VIS spectrum for HIPS was equal to that for ABS, and the UV/VIS spectrum of HDPE was equal to that for LDPE.

In general, samples with stronger UV absorption show smaller penetration depth and therefore smaller ablation craters. ${ }^{33,37}$ However, in the case of polymers not only the absorption of UV light but also their thermal properties and the ablation products' influence on the ablation crater volume have to be considered. Pham et $a l^{38}$ have shown a decrease in the ablation threshold with decreased glass transition temperatures. Kunz et al. raised the UV absorbance of polyestercarbonate samples through increased ester parts. The stronger UV absorbance resulted in higher ablation rates due to the increased release of gaseous decomposition products. ${ }^{39}$

The more pronounced absorption of UV light by polymers with aromatic groups in their structure resulted with the exception of PPA and PET in lower ablation crater volumes when compared to polymers without aromatic groups in their structure (Fig. 3). The largest ablation crater volume (POM) was four times larger than the least ablation crater volume (PEEK). Although PA 12 showed stronger UV absorbance the ablation

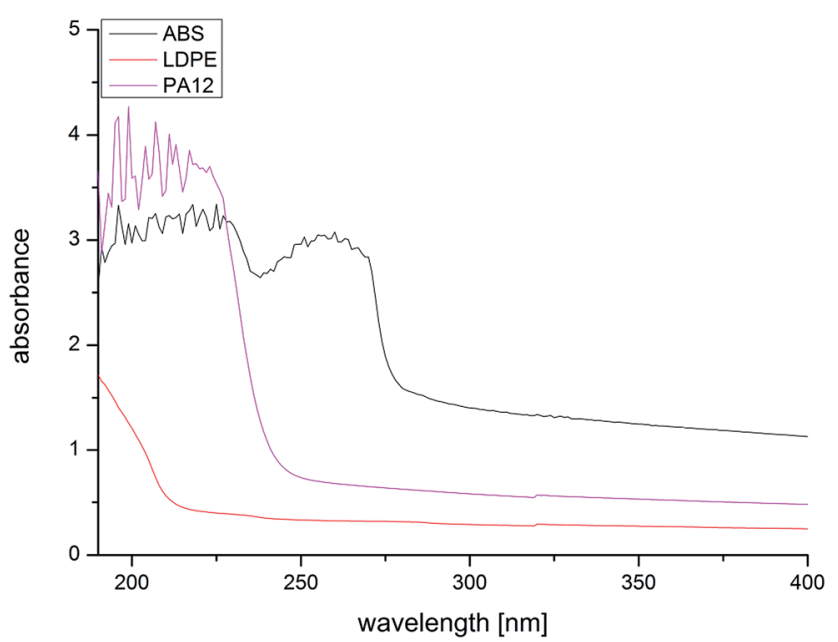

Fig. 2 UV/VIS spectra for $10 \mu \mathrm{m}$ sections of LDPE, PA 12 and ABS from $190 \mathrm{~nm}$ to $400 \mathrm{~nm}$. craters of the polyamides were comparable or even larger than those of the polyolefins.

Due to the weak absorption of UV light by the polyolefins, the energy from the laser penetrates deeper into the polymer leading to a higher heating of the material. The higher temperature within the polymer leads to a higher degree of bond breakage. This results in the ejection of more material, and in deeper but less smooth ablation craters. The less smooth ablation craters were also detected for other weakly absorbing samples..$^{33,37}$

Besides the difference in the polymeric structure, resulting in different UV absorption, also the thermal properties change. Especially the glass transition temperature is a critical factor. The glass transition temperatures of the polyolefins and POM show values of $-100{ }^{\circ} \mathrm{C}(\mathrm{LDPE})$ to $-10{ }^{\circ} \mathrm{C}(\mathrm{PP})$, by far the lowest compared to those of the other polymers used in this study. The glass transition temperatures of polymers with aromatic structures are between $47{ }^{\circ} \mathrm{C}$ (PBT) and $225{ }^{\circ} \mathrm{C}$ (PES) and those for polyamides are between $37^{\circ} \mathrm{C}$ (PA 12) and 50-60 ${ }^{\circ} \mathrm{C}$ (PA 6/PA 66). Samples with low glass transition temperatures show better flexibility in the polymer chains when compared to samples with higher glass transition temperatures. Due to the better flexibility less energy is required to break the bonds and to start the ablation.

Hence, the formation of comparable ablation crater volumes requires less energy for polymers with lower glass transition temperatures. For ABS and PA 12 equal UV absorption was found but the ablation crater volume of PA 12 was about 3.5 times greater than the ablation crater volume of ABS. The glass transition temperature of PA 12 is $37^{\circ} \mathrm{C}$ and that for ABS is $110{ }^{\circ} \mathrm{C}$. Since less energy is required for the ablation of PA 12 compared to ABS the ablation under the same conditions results in greater ablation crater volumes for PA 12. Due to the low glass transition temperature of LDPE even less energy is required for the ablation. Although this should result in an even deeper ablation crater for LDPE the ablation crater of PA 12 is about 2 times greater than that of LDPE. This probably can be attributed to the increased formation of gaseous decomposition

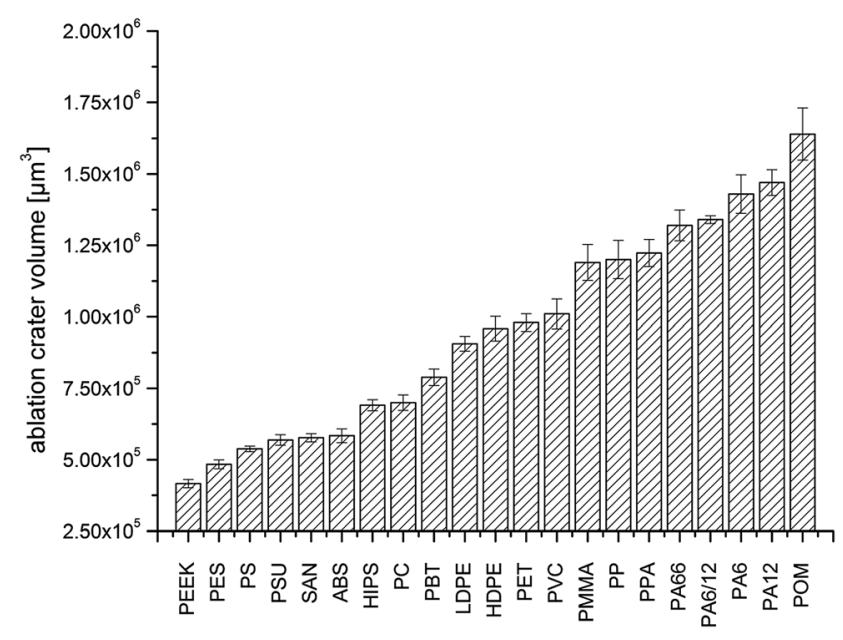

Fig. 3 Ablation crater volumes for twenty-one polymers applying 100 laser shots. 

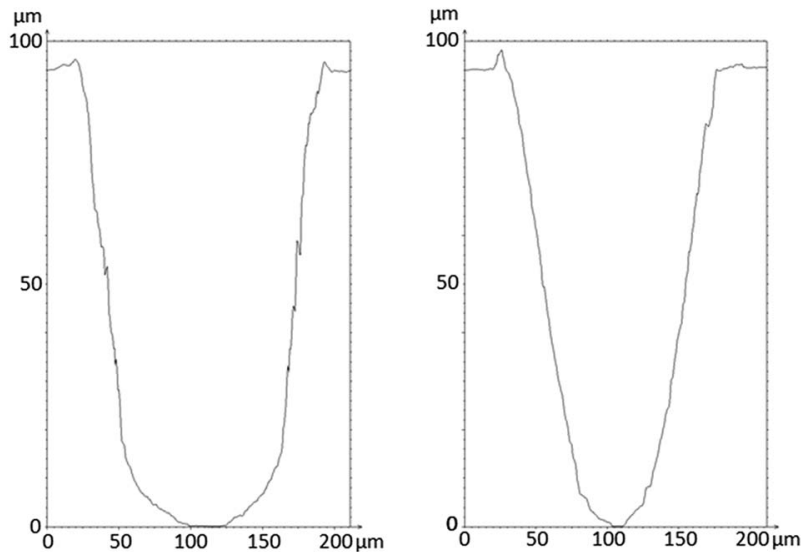

Fig. 4 Profile of a LDPE ablation crater; left: $Y$-direction in ablation gas flow; right: horizontal to ablation gas flow.

products. The decomposition of polyamides at higher temperatures results in the release of gaseous components like $\mathrm{CO}$, $\mathrm{CO}_{2}$ and $\mathrm{H}_{2} \mathrm{O} .{ }^{40,41}$ The detailed investigation of the kind of ablation product was not the aim of this work and has to be analyzed in greater detail in further studies.

The difference in the glass transition temperature not only influences the ablation crater volume but also the ablation crater shape. In the cases of LDPE, HDPE, PP and POM, which have the lowest glass transition temperatures, the material, especially on the walls of the ablation crater, melts to a higher degree than that in the cases of other polymers. Thus, the ablation performance is different for polymers with low glass transition temperatures since the higher flexibility of the polymer chains of these polymers leads to an easier set-back of the molten material into the ablation crater. This results in elongated shaped ablation craters as observed for LDPE for example (Fig. 4 and 6). The profiles of the resulting ablation craters are influenced by the ablation gas flow. In the direction of the ablation gas and horizontal to it the ablation crater shows a conical shape. However, in the $Y$-direction (direction of ablation gas flow) it is less pronounced compared to that in the $X$-direction (horizontal to the ablation gas flow). The ablation gas transports the molten material out of the ablation crater. Horizontally to the ablation gas flow the molten material can more easily set back into the ablation crater forming a more pronounced conical structure. Polymers with higher glass transition temperatures do not tend to melt at a high degree. The profiles in the $Y$-direction (direction of ablation gas flow) are comparable to the profiles in the $X$-direction (horizontal to ablation gas flow) as observed for PA 12 for example (Fig. 5). The upper parts of the PA 12 ablation crater are cylindrical in both profiles. The lower parts also show differences. The profile horizontal to the ablation gas flow shows a conical shape while a cylindrical shape is more pronounced in the direction of the ablation gas flow. Also polymers with higher glass transition temperatures tend to melt but to a much lower degree compared to polymers with very low glass transition temperatures.
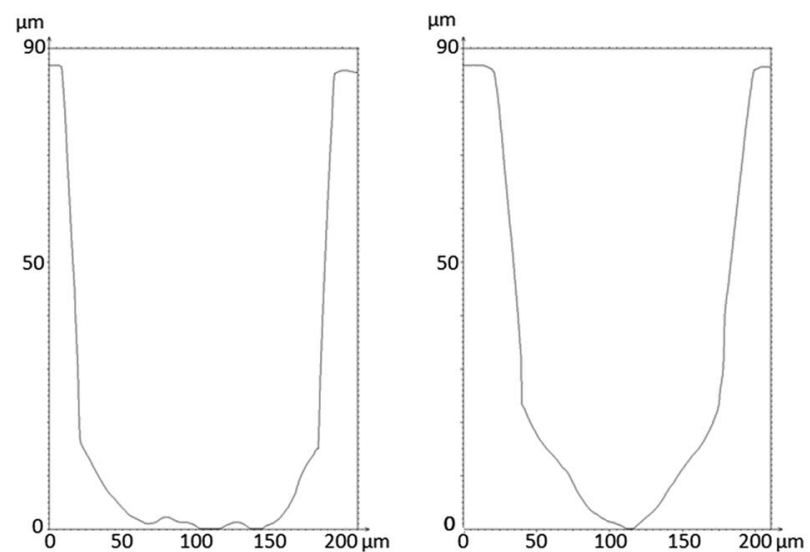

Fig. 5 Profile of a PA 12 ablation crater; left: $Y$-direction in ablation gas flow; right: horizontal to ablation gas flow.

The different ablation behaviors of LDPE, HDPE, PP and POM compared to those of other polymers were also observed through consideration of the surrounding surface of the ablation craters. The survey with a confocal microscope not only enables the measurements of ablation crater volume and form, but furthermore obtains information about the volume of deposited materials on the surfaces of the polymers.

The ablation of the aliphatic polymers LDPE, HDPE, PP and POM led to deposits of molten materials on the surfaces (Fig. 6). This also indicates that these polymers melt at a higher degree than that in the case of the other polymers during laser ablation. The formation of conical ablation craters caused by a setback of the molten material furthermore is confirmed by the deposited material on the surfaces of aliphatic polymers. Under the applied conditions deposits on the surfaces of approx. $2 \%$ of the ablation crater volume were found.

Polyolefins and POM exhibited a different behavior compared to the other polymers ablated. The products formed during the ablation of polyolefins can condense more easily and redeposit on the polymer surface. ${ }^{25}$ During the ablation of POM the formation of larger particles, which can easily condense and redeposit on the polymer surface, is very likely.

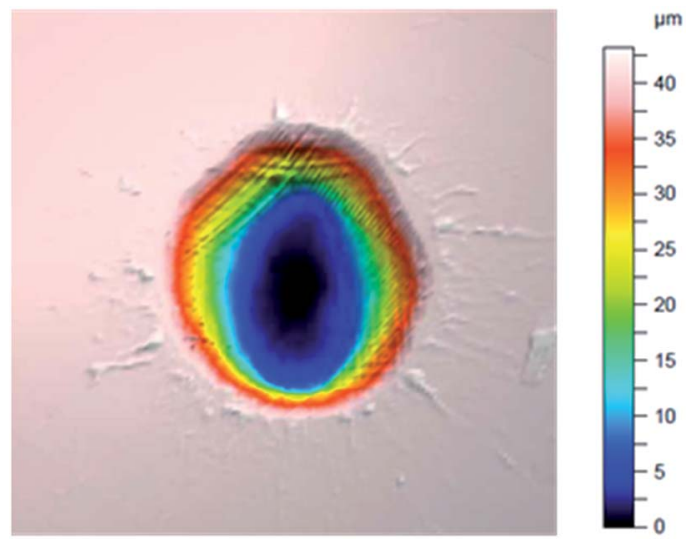

Fig. 6 3D plot of LDPE ablation crater and deposits on the polymer surface (100 laser shots). 


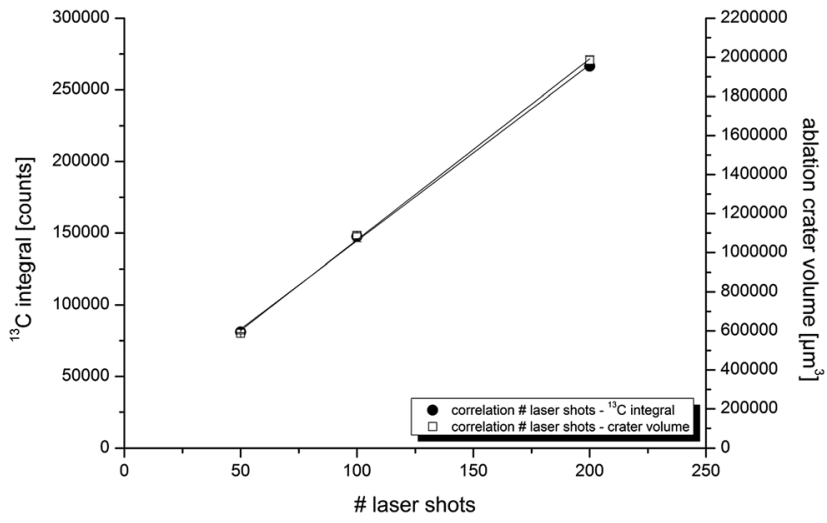

Fig. 7 Linear correlation for number of laser shots and ${ }^{13} \mathrm{C}$ integral (primary $\mathrm{Y}$-axis) and for number of laser shots and ablation crater volume (secondary $Y$-axis) observed for PA 6/12.

\section{Correlation of integrated carbon signal and ablated carbon}

In a first experiment the coherence between varying numbers of laser shots and the integrated ${ }^{13} \mathrm{C}$ signal intensity as well as the ablation crater volume was determined. Due to the optical limits of the applied confocal microscope set-up, the bottoms of the ablation craters of 200 laser shots of PMMA, PP, and POM were too deep in order to perform reliable confocal microscope measurements.

For polymers offering measurable ablation crater bottoms for 50, 100 and 200 laser shots, either a linear or a polynomial increase of the integrated ${ }^{13} \mathrm{C}$ signal curve was observed. These findings correlated well with the determined crater volumes. In the case of a linear increase of the integrated ${ }^{13} \mathrm{C}$ signal the ablation crater volume also showed a linear behavior (Fig. 7). For a polynomial increase of the integrated ${ }^{13} \mathrm{C}$ signal, a polynomial increase of the ablation crater volume was observed as well (Fig. 8).

An influence of the polymer structure on either a linear or polynomial increase of integrated ${ }^{13} \mathrm{C}$ signal and crater volume was observed. The structure of the polymeric backbone not only influences the ablation crater structure but also the increase of the ${ }^{13} \mathrm{C}$ signal intensity. Therefore a first direct coherence between the ablation crater volume and hence the ablated carbon and the measured ${ }^{13} \mathrm{C}-\mathrm{ICP}-\mathrm{MS}$ signal was determined.

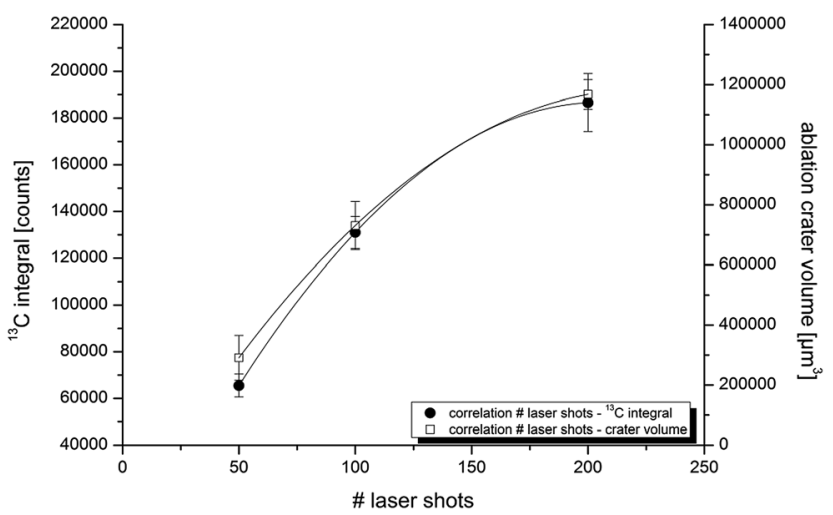

Fig. 8 Polynomial correlation for number of laser shots and ${ }^{13} \mathrm{C}$ integral (primary $\mathrm{Y}$-axis) and for number of laser shots and ablation crater volume (secondary $\mathrm{Y}$-axis) observed for PET.

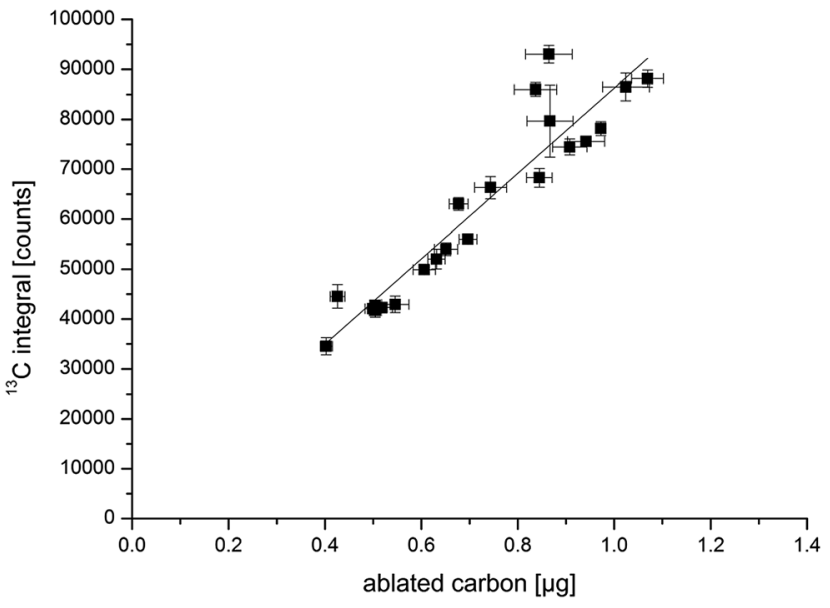

Fig. 9 Linear correlation of ${ }^{13} \mathrm{C}$ signal integral and ablated carbon for 21 polymers ablated in a He atmosphere applying 100 laser shots with a coefficient of determination of $R^{2}=0.885$.

It was found that the increase of the integrated ${ }^{13} \mathrm{C}$ signal as well as the increase in crater volume were related to the structure of the ablation crater. For ablation craters with a pronounced cylindrical shape, the increase was linear. The less pronounced cylindrical shaped ablation craters resulted in a polynomial increase in ${ }^{13} \mathrm{C}$ signal and crater volume.

The calculated ablated carbon and integrated ${ }^{13} \mathrm{C}$ signal intensities of seven different polymers were correlated choosing only those polymers with measurable ablation craters for 50 , 100 and 200 laser shots. Satisfactory coefficients of determination of $R^{2}=0.896$ (50 laser shots per point), $R^{2}=0.833$ (100 laser shots per point) and $R^{2}=0.930$ (200 laser shots per point) were obtained.

Since crater volumes for 100 laser shots were measureable for the first ten chosen polymers, all twenty-one polymers were ablated. The ablation crater volumes were measured followed by a correlation of the mean of the integrated ${ }^{13} \mathrm{C}$ signal intensities with the calculated total amount of carbon ablated which resulted in a coefficient of determination of $R^{2}=0.885$ (Fig. 9). The measurements have shown an existing correlation between ablated carbon and integrated ${ }^{13} \mathrm{C}$ signal intensity. This correlation is independent of the chemical compositions of the different polymers.

\section{Conclusions}

In this laser ablation study twenty-one different virgin polymers were investigated to establish internal standardization using the ${ }^{13} \mathrm{C}$ ICP-MS signal. It was shown that the kind of polymer substrate material has a strong physical effect on the ablation crater profile and ablated volume.

The correlation between ablated carbon by LA and the ${ }^{13} \mathrm{C}$ signal measured by ICP-MS was investigated to correct for changes in the ablation rates of different polymers.

Some effects caused by ablation, such as interaction of the laser with the sample and the resulting crater shape can be corrected using the ${ }^{13} \mathrm{C}$ signal. 
Nonetheless, the best means for quantification is a matrix matched standard reference material. Only if such a material is unavailable should a correction using the ${ }^{13} \mathrm{C}$ signal be applied for different polymers. Some effects caused by ablation, such as interaction of the laser with the sample and the resulting crater shape, are visible in the ion intensity as well. Differences in the ablated mass can be corrected. However, the influence of inorganic additives on the ablation crater shapes and volumes of different polymers must be investigated in further studies. Due to differences in the absorption properties of UV light of inorganic additives, an influence on the ablation craters is very likely.

\section{Acknowledgements}

The authors would like to thank Julia Deiters and Oliver Hagedorn (Westfälische Hochschule University of Applied Sciences, Bocholt, Germany) and Mario Adam (NanoFocus AG, Oberhausen, Germany) for their support with confocal microscope measurements. Many thanks to Cara Stough for reading the manuscript and her help to improve the language. This work was financially supported by the German Federal Ministry of Education and Research under the project number 17023X10.

\section{Notes and references}

1 S. Giacoppo, M. Galuppo, R. S. Calabro, G. D'Aleo, A. Marra, E. Sessa, D. G. Bua, A. G. Potorti, G. Dugo, P. Bramanti and E. Mazzon, Biol. Trace Elem. Res., 2014, 161, 151-160.

2 A. L. Lusher, M. McHugh and R. C. Thompson, Mar. Pollut. Bull., 2013, 67, 94-99.

3 N. Jakubowski, T. Prohaska, F. Vanhaecke, P. H. Roos and T. Lindemann, J. Anal. At. Spectrom., 2011, 26, 727-757.

4 K. D. Besecker, C. B. Rhoades Jr, B. T. Jones and K. W. Barnes, At. Spectrosc., 1998, 19, 193-197.

5 H. M. Kuss, Fresenius. J. Anal. Chem., 1992, 343, 788-793.

6 F. E. Smith and E. A. Arsenault, Talanta, 1996, 43, 1207-1268.

7 A. Wegner, C. Simons, S. Hanning, C. Mans and M. Kreyenschmidt, LaborPraxis, 2010, 34, 48-50.

8 A. M. Dobney, A. J. G. Mank, K. H. Grobecker, P. Conneely and C. G. de Koster, Anal. Chim. Acta, 2000, 423, 9-19.

9 R. E. Russo, X. Mao, H. Liu, J. Gonzalez and S. S. Mao, Talanta, 2002, 57, 425-451.

10 C. Simons, S. Hanning, A. Wegner, C. Mans, A. Janssen, M. Kreyenschmidt and J. A. C. Broekaert, J. Anal. At. Spectrom., 2008, 23, 1038-1041.

11 H. P. Longerich, D. Gunther and S. E. Jackson, Fresenius. J. Anal. Chem., 1996, 355, 538-542.

12 I.-A. Paun, A. Selimis, G. Bounos, G. Kecskemeti and S. Georgiou, Appl. Surf. Sci., 2009, 255, 9856-9860.

13 M. Hemmerlin and J. M. Mermet, Spectrochim. Acta, Part B, 1997, 52B, 1687-1694.

14 M. Motelica-Heino, O. F. X. Donard and J. M. Mermet, J. Anal. At. Spectrom., 1999, 14, 675-682.
15 J. L. Todoli and J. M. Mermet, Spectrochim. Acta, Part B, 1998, 53B, 1645-1656.

16 P. Arrowsmith and S. K. Hughes, Appl. Spectrosc., 1988, 42, 1231-1239.

17 T. Dumont, R. Bischofberger, T. Lippert and A. Wokaun, Appl. Surf. Sci., 2005, 247, 115-122.

18 M. Resano, E. Garcia-Ruiz and F. Vanhaecke, Spectrochim. Acta, Part B, 2005, 60B, 1472-1481.

19 C. Austin, F. Fryer, J. Lear, D. Bishop, D. Hare, T. Rawling, L. Kirkup, A. McDonagh and P. Doble, J. Anal. At. Spectrom., 2011, 26, 1494-1501.

20 J. Marshall, J. Franks, I. Abell and C. Tye, J. Anal. At. Spectrom., 1991, 6, 145-150.

21 I. De Schrijver, M. Aramendia, L. Vincze, M. Resano, A. Dumoulin and F. Vanhaecke, Spectrochim. Acta, Part B, 2007, 62B, 1185-1194.

22 M. Resano, F. Vanhaecke, D. Hutsebaut, K. De Corte and L. Moens, J. Anal. At. Spectrom., 2003, 18, 1238-1242.

23 B. Jackson, S. Harper, L. Smith and J. Flinn, Anal. Bioanal. Chem., 2006, 384, 951-957.

24 D. A. Frick and D. Guenther, J. Anal. At. Spectrom., 2012, 27, 1294-1303.

25 G. B. Blanchet, J. Appl. Phys., 1996, 80, 4082-4089.

26 S. F. Durrant, Fresenius. J. Anal. Chem., 1993, 347, 389-392.

27 K. C. Yung and D. W. Zeng, Surf. Coat. Technol., 2001, 145, 186-193.

28 S. Lazare and V. Granier, J. Appl. Phys., 1988, 63, 2110-2115. 29 J. S. Becker, R. C. Dietrich, A. Matusch, D. Pozebon and V. L. Dressler, Spectrochim. Acta, Part B, 2008, 63B, 12481252.

30 M. Kreyenschmidt, Application: WO Pat., 2006-DE1970 2007056977, 2007.

31 J. E. Andrew, P. E. Dyer, D. Forster and P. H. Key, Appl. Phys. Lett., 1983, 43, 717-719.

32 S. M. Eggins, L. P. J. Kinsley and J. M. G. Shelley, Appl. Surf. Sci., 1998, 127-129, 278-286.

33 P. Weis, H. P. Beck and D. Guenther, Anal. Bioanal. Chem., 2005, 381, 212-224.

34 Y. Li and Y. Zou, Adv. Mater., 2008, 20, 2952-2958.

35 G. Scheibe and R. Fauss, Kolloid-Z., 1952, 125, 139-149.

36 R. Srinivasan, Science, 1986, 234, 559-565.

37 K. Ito and M. Moriyasu, Microelectron. Eng., 1994, 25, 305312.

38 D. Pham, L. Tonge, J. Cao, J. Wright, M. Papiernik, E. Harvey and D. Nicolau, Smart Mater. Struct., 2002, 11, 668-674.

39 T. Kunz, J. Stebani, J. Ihlemann and A. Wokaun, Appl. Phys. A: Mater. Sci. Process., 1998, 67, 347-352.

40 B. G. Achhammer, F. W. Reinhart and G. M. Kline, J. Res. Natl. Bur. Stand., 1951, 46, 391-421; Research Paper No 2210.

41 C. L. Beyler and M. M. Hirschler, SFPE handbook of fire protection engineering, 2002, vol. 2, pp. 110-131. 